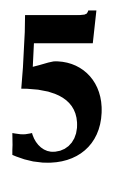

\title{
LA ADOLESCENCIA ANTE LA VIOLENCIA DE GÉNERO 2.0: CONCEPCIONES, CONDUCTAS Y EXPERIENCIAS
}

\author{
(ADOLESCENCE AND GENDER VIOLENCE 2.0: CONCEPTS, BEHAVIOR AND \\ EXPERIENCES)
}

Trinidad Donoso Vázquez

M. José Rubio Hurtado

Ruth Vilà Baños

Universidad de Barcelona

DOI: $10.5944 / e d u c X X 1.20180$

Cómo referenciar este artículo/How to reference this article:

Donoso Vázquez, T.; Rubio Hurtado, M. J. y Vilà Baños, R. (2018). La adolescencia ante la violencia de género 2.0: Concepciones, conductas y experiencias. Educación XX1, 21(1), 109-134, doi: 10.5944/educXX1.20180

Donoso Vázquez, T.; Rubio Hurtado, M. J. \& Vilà Baños, R. (2018). La adolescencia ante la violencia de género 2.0: Concepciones, conductas y experiencias. [Adolescence and gender violence 2.0: concepts, behavior and experiences]. Educación XX1, 21(1), 109-134, doi: 10.5944/educXX1.20180

\section{RESUMEN}

El objetivo del estudio es presentar un instrumento de medida sobre las violencias de género 2.0 y analizar las percepciones y experiencias de adolescentes, así como las respuestas ante tales violencias. Se ha realizado un estudio por encuesta a 3.043 adolescentes de Cataluña, Aragón, Galicia, Andalucía, Islas Baleares y Canarias. Entre los resultados encontrados destaca que los y las adolescentes creen que hay más violencia online que offline; y perciben las acciones más directas y evidentes ligadas a la violencia sexual, pero en cambio las conductas que menos se perciben como violentas son aquellas en las que la mujer es tratada como objeto sexual, y especialmente no se perciben como violentas las conductas de control que se ejercen sobre la pareja a través de los entornos virtuales. La tendencia a la agresión es masculina, pero las chicas muestran más conductas agresoras en violencias relacionados con los mitos del amor romántico. Las respuestas de las y los adolescentes son pasivas ante las violencias de género 2.0, aunque las chicas superan a los chicos en las respuestas activas frente a la violencia. Se concluye con la necesidad de realizar intervenciones educativas para preparar a las y los adolescentes ante las violencias de género 2.0. Intervenciones que deberán estar orientadas a: deconstruir los mitos del amor romántico que sustentan falsas ideas sobre 
la confianza en la pareja, a concienciar a las chicas sobre su mayor vulnerabilidad en las redes sociales, a explicar a los y las adolescentes los riesgos de todas las redes sociales, y a alentar a los jóvenes a denunciar las acciones de violencia e implicar a la familia y la escuela en estos asuntos.

\section{PALABRAS CLAVE}

Género; violencia; adolescencia; internet; redes sociales.

\section{ABSTRACT}

The aim of this study is to present a measurement instrument for gender violence 2.0 and analyze the perceptions and experiences of adolescents, and their responses to such violence. A study survey of 3,043 teens from Catalonia, Aragon, Galicia, Andalusia, the Balearics and the Canaries was undertaken. Among the results, we found that adolescents believe that there is more violence online than offline. They are aware of the most direct and obvious actions related to sexual violence, however behavior that is less frequently perceived to be violent is that where the woman is treated as a sexual object, and in particular, controlling online behavior against the girlfriend or boyfriend is not considered to be violent. The tendency for aggression is male, but girls show more aggressive behaviors related to myths of romantic love. The responses of the adolescents are passive towards gender violence 2.0, though girls outperform boys in active responses to violence. It concludes with the need for educational interventions to prepare the adolescents for gender violence 2.0. Interventions should be designed to deconstruct the myths of romantic love that support misconceptions about the trust between partners, to educate girls about their increased vulnerability on social networks, to explain to adolescents all of the risks on social networks and to encourage young people to report acts of violence and to involve their family and school in these matters.

\section{KEYWORDS}

Gender; violence; teenagers; internet; social networks.

\section{INTRODUCCIÓN}

Las nuevas tecnologías están propiciando nuevas formas de relación y socialización entre personas, algunas de ellas reflejo de la realidad no virtual y otras adaptadas a las nuevas herramientas que el espacio virtual ofrece. Estas nuevas formas de comunicación e interacción online se fundamentan en un espíritu democrático de compartir información y parecen tejerse con 
un velo de neutralidad y horizontalidad, en el que categorías sociales como la clase, el género, la etnia, etc. parecen difuminarse. Pueden constituirse en un medio de empoderamiento para las mujeres, para personas con dificultades, vulnerables, con diversidad funcional y para grupos discriminados, ya que les permite dar a conocer sus opiniones, y elaborar estrategias y medidas para incidir en la toma de decisiones (García Ramos, s/f). No obstante, el espacio virtual no es neutro como a priori podría pensarse y los datos así lo demuestran. Se convierte en muchas ocasiones en un espacio en el cual las personas quedan expuestas a agresiones que pueden ocasionar múltiples daños psicológicos que llevan a secuelas en la vida real y pueden tener repercusiones de largo alcance.

Si hablamos de cifras de agresiones en las redes en relación con la juventud, estas son muy variables. Según la revisión de estudios realizada por Calvete, Orue, Estévez, Villardón y Padilla (2010) la prevalencia del acoso online oscila entre un $1,7 \%$ y un $35,7 \%$. Una encuesta en la que participaron 25 países europeos (Garmendia, Garitaonandia, Martínez y Casado, 2011), concluyó que en España, el 15\% de los menores han sufrido acoso escolar online u offline, mientras que el $9 \%$ afirma haber acosado a otro menor. Si consideramos únicamente el bullying en Internet, el 5\% afirma haber recibido estos mensajes de acoso y el 3\% haberlos enviado. En el caso del envío de mensajes sexuales pasa algo parecido, el 7\% de menores españoles afirma haber recibido esos mensajes, pero únicamente el $2 \%$ afirma enviarlos.

En las agresiones a través de internet y del móvil es preciso distinguir entre las ciberagresiones o Cyberbullying, referido al acoso en línea de jóvenes y adolescentes (Patchin e Hinduja, 2006; Li, 2007), y las ciberagresiones en las que interviene la variable género. Cabe destacar que en ambos casos al término acoso se le atribuye la acepción moral de daño integral a la persona.

Cuando se aborda la violencia en línea desde esta última perspectiva, la del género, los estudios se centran bien en realizar segregaciones para diferenciar conductas de agresión o de victimización según el sexo (Calmaestra, 2011), bien en la óptica de las relaciones afectivas. En ambos casos los resultados de las investigaciones sitúan a las mujeres en una posición de vulnerabilidad frente a los hombres.

La proporción de niños que participan como agresores es mayor, al igual que la proporción de niñas que participan como víctimas. Según la publicación que realizó la organización WHOA (2012), un 80\% de las víctimas de acoso a través de la red fueron mujeres y dos tercios de los agresores fueron hombres. Desde la óptica de las relaciones afectivas, la problemática se aborda atendiendo a la posición de hombres y mujeres en la estructura 
social y estableciendo las consecuencias con relación al establecimiento o subversión del orden cultural. «El ciberacoso en tanto que violencia de género [...] tiene como objetivo la dominación, la discriminación y, en definitiva, el abuso de la posición de poder donde el hombre acosador tiene o ha tenido alguna relación afectiva o de pareja con la mujer acosada» (Torres, 2013, p. 27). La investigación de Estébanez y Vázquez (2013) es un ejemplo de aplicación del enfoque de género en el análisis que hacen del ciberacoso. Dicha investigación se centra en jóvenes de entre 13 y 29 años y pone de manifiesto que las chicas son contactadas y acosadas por el único motivo de ser chicas. Y el mismo estudio concluye que la conducta de acoso genera en las chicas una respuesta inmediata, sea a través de borrar o no aceptar, pero no una mayor conciencia de lo que es la violencia machista.

El fundamento de la violencia de género son las relaciones asimétricas de poder encaminadas a establecer o perpetuar relaciones de desigualdad (Arisó y Mérida 2010). Este orden social imperante se traspasa a los espacios virtuales atribuyendo lugares determinados, específicos, constreñidos y subalternos a las mujeres y a otras personas que son discriminadas por transgredir las formas de conducta obligatorias que propone la sociedad patriarcal (homosexuales, transexuales, heterosexuales que se apartan de los dictámenes del patriarcado...).

Según Lagarde (1997) es la supremacía de los hombres y de lo masculino, sobre la interiorización de las mujeres y lo femenino. Es así mismo, un orden de dominio de unos hombres sobre otros y de enajenación de las mujeres. Las mujeres en distintos grados, son expropiadas y sometidas a opresión, de manera predeterminada. El mundo resultante es asimétrico, desigual, enajenado, de carácter androcéntrico, misógino y homófobo. La violencia está legitimada en el sistema patriarcal para mantener la jerarquización de lo masculino hacia lo femenino y la inferioridad de las mujeres, esta inferioridad se fundamenta en el sexismo o en la misoginia. Esta violencia también es ejercida cuando las personas se enfrentan a la ideología patriarcal.

En el estudio que se presenta en este artículo se plantea una propuesta diferente para el abordaje de la ciberviolencia en función del género, atendiendo a los motivos que originan las conductas (Donoso, Rubio y Vilà, 2017), para así comprobar cómo las relaciones marcadas por los roles de genero siguen perpetuándose en Internet a través de una estructura social jerarquizada y discriminatoria basada en el género, consolidando estereotipos y ejerciendo una violencia ideológica y simbólica que perpetúa un status quo de dominación hacia grupos vulnerables como son las mujeres y todas aquellas personas que se apartan de las imposiciones heteronormativas del patriarcado. Las conductas sobre violencias de género más habituales en los entornos virtuales que frecuentan las y los adolescentes y los motivos se resumen en la tabla 1. 


\section{Tabla 1}

\section{Conductas de violencias de género 2.0 y motivos (Donoso, Rubio y Vilà, 2017)}

\begin{tabular}{|c|c|}
\hline Conductas de violencias de género 2.0 & Motivos \\
\hline $\begin{array}{l}\text { Insultar por internet a una chica porque tiene varias } \\
\text { parejas, acosar a una chica porque es provocativa con el } \\
\text { móvil o redes sociales, meterse con una chica a través de } \\
\text { internet porque no se interesa por los chicos, insultar en } \\
\text { Internet a una chica por no haber tenido relaciones con } \\
\text { chicos, llamar por teléfono o enviar correos o mensajes } \\
\text { insistentes a una chica provocativa }\end{array}$ & $\begin{array}{l}\text { Apartarse de la normatividad } \\
\text { sexual femenina }\end{array}$ \\
\hline $\begin{array}{l}\text { Meterse con personas homosexuales o transexuales en } \\
\text { internet, difundir en internet la orientación sexual de al- } \\
\text { guien sin su permiso, insultar en la red a una persona por } \\
\text { ser o pensar que es homosexual o transexual, suplantar la } \\
\text { identidad de alguien haciéndole pasar por homosexual y } \\
\text { ridiculizarlo en internet o móvil, decir que homosexuales } \\
\text { o transexuales son «enfermos», ridiculizar a alguien por } \\
\text { su orientación sexual }\end{array}$ & $\begin{array}{l}\text { Transgredir heteronormatividad } \\
\text { sexual obligatoria }\end{array}$ \\
\hline
\end{tabular}

Colgar fotos o videos humillantes de chicas obesas o feas en internet o a través del móvil, mostrar a la mujer simplemente como un objeto sexual en alguna página web, participar en una Web donde se puntúa el físico de las chicas, insultar a una chica por tener un físico poco atractivo, meterse con un chico por tener un físico poco masculino, mostrar la foto de una chica simplemente como un objeto sexual en Facebook u otras redes sociales
Amenazar a una chica para mantener una relación de pareja, llenar el correo de alguien con contenido sexual, conseguir fotos de una persona para chantajearla para aprovecharse sexualmente de ella, difundir videos/fotos sexis de una chica en internet sin su permiso, enviar imá- genes con contenido sexual de una persona sin su consen- timiento, engañar o chantajear a una persona utilizando material online para tener relaciones sexuales con ella, difundir fotos o vídeos de contenido erótico, pornográfico o sexual, sin permiso

\begin{abstract}
Revisar varias veces por internet o móvil dónde está su pareja y qué hace, obligar a la novia a borrar algunos amigos en Facebook u otra red social, pedir a la pareja las claves de acceso de su correo electrónico u otras cuentas de internet, controlar a la pareja en Facebook oTwitter, conocer la contraseña de la pareja en las redes sociales para bloquear amistades, coger el móvil de la pareja para ver llamadas e inspeccionar, obligar a la pareja a quitar fotos de amigos en Facebook o dejar de chatear con alguien
\end{abstract}

Meterse con alguien en internet por su ideología feminista, echar a alguien de un chat o foro por el hecho de ser mujer, enviar imágenes o hacer chistes sobre agresiones contra las mujeres...
Imposiciones del canon de belleza heteronormativo Violencia asociada a mitos amor
romántico

Otros motivos varios: violencia por ideología, etc. 
En este artículo se proponen los siguientes objetivos de estudio:

- Presentar un instrumento de medida sobre las violencias de género 2.0 en la adolescencia.

- Analizar las percepciones de los y las adolescentes sobre Internet y telefonía móvil y la violencia de género 2.0.

- Analizar las experiencias que han tenido los y las adolescentes, en cuanto a ciberagresiones, cibervictimización, y ciberobservación de la VG 2.0, así como las respuestas ante la misma cuando son víctimas u observadores.

La finalidad última de la investigación no es solo determinar la prevalencia del fenómeno de la violencia de género 2.0, sino que el conocimiento de esta sirva de diagnóstico para proponer pautas educativas acordes con los resultados encontrados para aplicarlas en acciones formativas dirigidas a alumnado de los centros de secundaria.

\section{Método}

Se ha realizado un estudio por encuesta usando como instrumento de recogida de datos un cuestionario para adolescentes de Educación Secundaria Obligatoria de las regiones de Cataluña, Aragón, Galicia, Andalucía, Islas Baleares y Canarias.

\section{Instrumento}

Para analizar las violencias de género desde la óptica de la heteronormatividad patriarcal se aplicó el Cuestionario de violencias de género 2.0 (Donoso et al., 2014a), al cual se respondió de forma online durante el periodo marzo-junio de 2015 en las diferentes regiones que forman parte del estudio.

Las dimensiones del Cuestionario de ciberviolencias de género se relacionan con los siguientes aspectos teóricos:

- Aspectos relacionados con el objeto de estudio: competencias y uso de entornos digitales, percepción de internet como entorno violento, percepción de impunidad de la red, percepción de tener características personales de vulnerabilidad.

- Concepciones sobre las violencias de género que surgen de la normatividad patriarcal hacia hombres y mujeres (medidas mediante escala Likert de cinco niveles de respuesta). 
- Experiencias sufridas en los entornos virtuales que surgen de la normatividad patriarcal hacia hombres y mujeres: referida al hecho de padecer, ejercer u observar violencia de género 2.0. (medidas mediante escala Likert de cinco niveles de respuesta).

- Respuestas y formas de actuar cuando se sufre o se observan conductas violencia de género 2.0.

Las escalas del cuestionario muestran fiabilidad en relación con el índice de consistencia interna Alpha de Cronbach (tabla 2) y presentan validez de contenido (Donoso et al, 2014b) y validez ligada al criterio en la escala de experiencias como agresor, que correlaciona positivamente $(\mathrm{r}=0,638$, $\mathrm{p}=0,00)$ con la escala de Cyberbullying offending de Hinduja y Patchin (2011), la cual está compuesta por 9 ítems orientados a conocer la agresión a través de Internet y el móvil (alpha de Crombach de 0.788).

Tabla 2

Dimensiones del Cuestionario de ciberviolencias de género y fiabilidad de las escalas

\begin{tabular}{|c|c|c|c|}
\hline Dimensiones & Categorías de análisis & N. ${ }^{o}$ de ítems & \multirow{6}{*}{$\begin{array}{l}\text { Alpha de } \\
\text { Cronbach }\end{array}$} \\
\hline \multirow{5}{*}{ Caracterización } & Datos sociodemográficos & 7 & \\
\hline & Perfil de dominio y uso tecnológico & 15 & \\
\hline & $\begin{array}{l}\text { Percepción de internet como entor- } \\
\text { no violento }\end{array}$ & 16 & \\
\hline & Percepción de impunidad de la red & 6 & \\
\hline & $\begin{array}{l}\text { Percepción de tener características } \\
\text { personales de vulnerabilidad }\end{array}$ & 2 & \\
\hline \multirow{6}{*}{$\begin{array}{l}\text { Concepto de V. G. } 2.0 \\
\text { Escala Likert-5 }\end{array}$} & $\begin{array}{l}\text { Apartarse de la normatividad sexual } \\
\text { femenina }\end{array}$ & 3 items & $\square=0.772$ \\
\hline & $\begin{array}{l}\text { Transgredir heteronormatividad } \\
\text { sexual obligatoria }\end{array}$ & 3 items & $\square=0.798$ \\
\hline & $\begin{array}{l}\text { Imposiciones del canon de belleza } \\
\text { heteronormativo }\end{array}$ & 3 items & $\square=0.744$ \\
\hline & Violencia sexual & 3 items & $\square=0.838$ \\
\hline & $\begin{array}{l}\text { Violencia asociada a mitos amor } \\
\text { romántico }\end{array}$ & 3 items & $\square=0.818$ \\
\hline & Total escala concepto de VG 2.0 & 15 items & $\square=\mathbf{0 . 9 3 1}$ \\
\hline
\end{tabular}




\begin{tabular}{|c|c|c|c|}
\hline Dimensiones & Categorías de análisis & N. ${ }^{o}$ de ítems & \\
\hline & $\begin{array}{l}\text { Apartarse de la normatividad sexual } \\
\text { femenina }\end{array}$ & 4 items & $\square=0.621$ \\
\hline & $\begin{array}{l}\text { Transgredir heteronormatividad } \\
\text { sexual obligatoria }\end{array}$ & 4 items & $\square=0.760$ \\
\hline $\begin{array}{l}\text { Ciberagresiones } \\
\text { en VG } 2.0\end{array}$ & $\begin{array}{l}\text { Imposiciones del canon de belleza } \\
\text { heteronormativo }\end{array}$ & 4 items & $\square=0.659$ \\
\hline \multirow[t]{4}{*}{ Escala Likert-3 } & Violencia sexual & 4 items & $\square=0.753$ \\
\hline & $\begin{array}{l}\text { Violencia asociada a mitos amor } \\
\text { romántico }\end{array}$ & 4 items & $\square=0.776$ \\
\hline & Otras conductas & 3 items & \\
\hline & Total escala ciberagresión & 23 items & $\square=\mathbf{0 . 9 0 1}$ \\
\hline \multirow{7}{*}{$\begin{array}{c}\text { Ciberavictimizaciones } \\
\text { en VG } 2.0 \\
\text { Escala Likert-3 }\end{array}$} & $\begin{array}{l}\text { Apartarse de la normatividad sexual } \\
\text { femenina }\end{array}$ & 4 items & $\square=0.772$ \\
\hline & $\begin{array}{l}\text { Transgredir heteronormatividad } \\
\text { sexual obligatoria }\end{array}$ & 4 items & $\square=0.777$ \\
\hline & $\begin{array}{l}\text { Imposiciones del canon de belleza } \\
\text { heteronormativo }\end{array}$ & 4 items & $\square=0.610$ \\
\hline & Violencia sexual & 4 items & $\square=0.765$ \\
\hline & $\begin{array}{l}\text { Violencia asociada a mitos amor } \\
\text { romántico }\end{array}$ & 4 items & $\square=0.860$ \\
\hline & Otras conductas & 3 items & \\
\hline & Total escala cibervictimización & 23 items & $\square=\mathbf{0 . 9 1 2}$ \\
\hline \multirow{7}{*}{$\begin{array}{c}\text { Ciberobservaciones } \\
\text { de VG } 2.0 \\
\text { Escala Likert-3 }\end{array}$} & $\begin{array}{l}\text { Apartarse de la normatividad sexual } \\
\text { femenina }\end{array}$ & 4 items & $\square=0.840$ \\
\hline & $\begin{array}{l}\text { Transgredir heteronormatividad } \\
\text { sexual obligatoria }\end{array}$ & 4 items & $\square=0.836$ \\
\hline & $\begin{array}{l}\text { Imposiciones del canon de belleza } \\
\text { heteronormativo }\end{array}$ & 4 items & $\square=0.838$ \\
\hline & Violencia sexual & 4 items & $\square=0.845$ \\
\hline & $\begin{array}{l}\text { Violencia asociada a mitos amor } \\
\text { romántico }\end{array}$ & 4 items & $\square=0.929$ \\
\hline & Otras conductas & 3 items & \\
\hline & Total escala ciberobservación & 23 items & $\square=\mathbf{0 . 9 6 1}$ \\
\hline $\begin{array}{c}\text { Respuestas ante la } \\
\text { violencia de género } 2.0\end{array}$ & $\begin{array}{l}\text { Respuestas como víctima } \\
\text { Respuestas como observador/a }\end{array}$ & & \\
\hline
\end{tabular}

\section{Participantes}

La selección de la muestra fue mediante muestreo aleatorio por conglomerados, siendo los conglomerados los centros de secundaria de cada 
región participante en el estudio. A los centros se accedió mediante contacto personal con el equipo directivo. Del total de estudiantes incluidos en la muestra, contestaron finalmente 3.043 adolescentes, considerando un nivel de confianza del $95,5 \%$ para poblaciones infinitas, donde p y $q=0.5$, lo que supone un margen de error de \pm 0.018 .

Los adolescentes están escolarizados en $3 .^{\circ}$ y $4 .^{\circ}$ de ESO y pertenecen a 6 comunidades autónomas del estado español: Baleares (25\%), Catalunya (24\%), Galicia (21\%), Andalucía (12\%), Aragón (9\%) y Canarias (9\%). La muestra presenta una media de edad de 15 años, con porcentaje similar de chicas y chicos ( $52 \%$ y $48 \%$ respectivamente). El $26 \%$ dice tener pareja afectiva y casi la mitad (48\%) cree tener alguna característica de vulnerabilidad para poder padecer violencia. Mayoritariamente la muestra se considera heterosexual (89\%), aunque hay diversidad de opciones.

\section{Análisis de datos}

Se ha realizado un análisis descriptivo de todas las variables del estudio, en relación con las medidas de tendencia central y desviación en el caso de las variables cuantitativas y frecuencias de aparición de las categorías para las variables cualitativas.

También se han aplicado pruebas de contraste de hipótesis a fin de poder establecer diferencias entre ambos sexos en relación con las variables de interés consideradas en el estudio.

\section{RESULTADOS}

\section{Usos, riesgos y competencias tecnológicas}

Los recursos más utilizados por los y las adolescentes son whatsapp, instagram, y youtube en general: El 92\% dicen utilizar facebook cada día; el 61\%, instagram; y el 58\% dicen utilizar youtube cada día.

Los recursos en los que se percibe un mayor riesgo de sufrir violencia son facebook, whatsapp, ask.fm e instagram, recursos que permiten una interactividad, en cambio youtube destaca por ser percibido con menor riesgo. En general, los y las adolescentes perciben riesgo en algunas conductas online, entre la que destacan "poner información personal en la red» o "chatear repetidas veces con una persona de la cual no tienes indicios de quién es». Pero no perciben tan peligroso colgar fotos o videos personales. 
Por otro lado destaca que un porcentaje importante de adolescentes percibe que tiene competencias digitales relacionadas con la seguridad en la red, siendo la función más conocida la de bloqueo de personas y la más desconocida la de desactivación de la geolocalización del móvil, tal y como se aprecia en la tabla 3.

Tabla 3

Competencias digitales

\begin{tabular}{lcc}
\hline & Total & $\%$ \\
\hline $\begin{array}{l}\text { ¿Sabes bloquear a la gente que te molesta en entornos } \\
\text { virtuales? }\end{array}$ & 2984 & 98,1 \\
$\begin{array}{l}\text { ¿Sabes modificar las condiciones de privacidad de las } \\
\text { redes sociales que utilizas? }\end{array}$ & 2820 & 92,7 \\
$\begin{array}{l}\text { ¿Sabes desactivar la geolocalización de tu móvil para } \\
\text { que no puedan controlar dónde estás? }\end{array}$ & 2260 & 74,3 \\
$\begin{array}{l}\text { ¿Sabes denunciar las fotografías que se suben a la red } \\
\text { indebidamente? }\end{array}$ & 2734 & 89,8 \\
$\begin{array}{l}\text { ¿Abres correos electrónicos dudosos de procedencia } \\
\text { desconocida? }\end{array}$ & 709 & 23,3 \\
$\begin{array}{l}\text { ¿Tienes algún antivirus en el ordenador que te avisa o } \\
\text { bloquea las páginas peligrosas? }\end{array}$ & 2516 & 82,7 \\
\hline
\end{tabular}

\section{¿Existe impunidad en la red?}

Mayoritariamente la muestra estudiada cree que hay más violencia online que offline, el 90\% de las y los adolescentes así lo manifiestan. Además el $85 \%$ piensa que no se castiga en general a las personas que actúan de forma ilegal o dañina en la red. Ante esta situación, una gran proporción de adolescentes opina que es necesario que se intervenga ante los delitos online. Concretamente, más del $90 \%$ cree que debería intervenir la policía o un organismo especializado en violencia en Internet. En tercer lugar los adolescentes señalan la familia; y algo más de la mitad a las amistades. $\mathrm{El} 62 \%$ cree que la escuela no debería intervenir.

\section{Scale \\ Conductas de ciberacoso medidas con la Cyberbullying offending}

Aproximadamente 2 de cada 10 adolescentes han sido acosados o acosadas en entornos virtuales, y en menor medida manifiestan acosar (media de 11 puntos sobre 45). Destaca el tipo de acoso: «colgar comentarios crueles en internet» como aquel más elegido (tabla 4). 
Tabla 4

Estadisticos descriptivos de la Cyberbullying offending scale. Hinduja y Patchin (2011)

\begin{tabular}{lcc}
\hline \multicolumn{1}{c}{ Escala de $\mathbf{1}$ a 5 puntos } & Media & Desv. típ. \\
\hline $\begin{array}{l}\text { He acosado a otra persona por Internet o móvil } \\
\begin{array}{l}\text { He colgado en Internet comentarios crueles u ofensivos } \\
\text { sobre alguien }\end{array}\end{array}$ & 1,1735 &, 52872 \\
$\begin{array}{l}\text { He difundido rumores sobre alguien en Internet } \\
\text { He amenazado con hacer daño a alguien en Internet }\end{array}$ & 1,2721 &, 66437 \\
$\begin{array}{l}\text { He amenazado con hacer daño a alguien en un mensaje } \\
\text { de móvil }\end{array}$ & 1,2067 &, 59280 \\
$\begin{array}{l}\text { Me he hecho pasar por alguien y he actuado de forma cruel } \\
\text { u ofensiva }\end{array}$ & 1,2435 &, 61979 \\
$\begin{array}{l}\text { He colgado una imagen cruel u ofensiva de otra persona } \\
\text { He creado una página Web cruel u ofensiva sobre alguien }\end{array}$ & 1,1827 &, 53798 \\
$\begin{array}{l}\text { He colgado un vídeo cruel u ofensivo de alguien } \\
\text {, 477790 }\end{array}$ & 1,0292 &, 22382 \\
\hline
\end{tabular}

\section{Concepto de violencias de género 2.0}

Los y las adolescentes tienen una elevada conciencia de lo que es violencia de género en entornos virtuales, por lo tanto saben interpretar como violencia las manifestaciones de la misma (tabla 5).

Tabla 5

Estadísticos descriptivos de la Escala Concepto de VG 2.0

\begin{tabular}{lcc}
\hline \multicolumn{1}{c}{ Escala de 3 a 15 puntos } & Media & Desv. típ. \\
\hline Apartarse de la normatividad sexual femenina & 11,9244 & 2,77685 \\
Transgredir heteronormatividad sexual obligatoria & 12,8143 & 2,58581 \\
Imposiciones del canon de belleza heteronormativo & 12,6356 & 2,65758 \\
Violencia sexual & 13,4542 & 2,57844 \\
Violencia asociada a mitos amor romántico & 11,8853 & 3,04335 \\
\hline
\end{tabular}


Las conductas que menos se perciben como violentas son aquellas conductas en las cuales las chicas no se ajustan al patrón de sexualidad femenino marcado, como por ejemplo una chica que presuntamente tiene varias parejas, una chica provocativa o una chica que se aparta del canon de belleza femenino impuesto, ya sea porque su físico se aparta de la cultura de la hipersexualización o bien porque es tratada como objeto sexual en algunas páginas Webs. Por último, tampoco se percibe como conducta violenta el control que se ejerce a través de entornos virtuales a la pareja.

\section{Experiencias en violencias de género 2.0}

En general la muestra afirma que ha observado conductas violentas en mayor medida de lo que ha sido víctima o persona agresora (tal y como se deduce de la tabla 6).

Tabla 6

Puntuaciones en las escalas de experiencia en violencias de género 2.0

\begin{tabular}{|c|c|c|c|}
\hline & & Media & $\begin{array}{l}\text { Desviación } \\
\text { Típica }\end{array}$ \\
\hline \multirow{5}{*}{$\begin{array}{l}\text { Escala de Ciberagresiones } \\
\text { en VG } 2.0\end{array}$} & $\begin{array}{l}\text { Apartarse de la normatividad sexual } \\
\text { femenina }\end{array}$ & 1,21 & ,43 \\
\hline & $\begin{array}{l}\text { Transgredir heteronormatividad } \\
\text { sexual obligatoria }\end{array}$ & 1,12 & ,39 \\
\hline & $\begin{array}{l}\text { Imposiciones del canon de belleza } \\
\text { heteronormativo }\end{array}$ & 1,29 &, 52 \\
\hline & Violencia sexual & 1,08 & 32 \\
\hline & $\begin{array}{l}\text { Violencia asociada a mitos amor } \\
\text { romántico }\end{array}$ & 1,30 &, 58 \\
\hline \multirow{5}{*}{$\begin{array}{l}\text { Escala de Ciberavictimiza- } \\
\text { ciones en VG } 2.0\end{array}$} & $\begin{array}{l}\text { Apartarse de la normatividad sexual } \\
\text { femenina }\end{array}$ & 1,19 & ,46 \\
\hline & $\begin{array}{l}\text { Transgredir heteronormatividad } \\
\text { sexual obligatoria }\end{array}$ & 1,12 & ,40 \\
\hline & $\begin{array}{l}\text { Imposiciones del canon de belleza } \\
\text { heteronormativo }\end{array}$ & 1,22 & ,46 \\
\hline & Violencia sexual & 1,12 & 39 \\
\hline & $\begin{array}{l}\text { Violencia asociada a mitos amor } \\
\text { romántico }\end{array}$ & 1,31 & ,66 \\
\hline
\end{tabular}




\begin{tabular}{llcc}
\hline & & Media & $\begin{array}{c}\text { Desviación } \\
\text { Típica }\end{array}$ \\
\hline & $\begin{array}{l}\text { Apartarse de la normatividad sexual } \\
\text { femenina } \\
\text { Transgredir heteronormatividad } \\
\text { sexual obligatoria }\end{array}$ & 2,29 &, 97 \\
$\begin{array}{l}\text { Escala de Ciberobserva- } \\
\text { ciones de VG 2.0 }\end{array}$ & $\begin{array}{l}\text { Imposiciones del canon de belleza } \\
\text { heteronormativo } \\
\text { Violencia sexual } \\
\text { Violencia asociada a mitos amor } \\
\text { romántico }\end{array}$ & 2,39 & 1,00 \\
& & 2,34 &, 93 \\
& & 1,11 \\
\hline
\end{tabular}

Las agresiones se traducen en mayor medida en colgar fotos o videos humillantes de chicas obesas o feas, mostrar a la mujer simplemente como objeto sexual, meterse con un chico por tener un físico poco masculino y todas las conductas asociadas a los mitos del amor romántico. La victimización se da mayoritariamente en las mismas conductas de agresión. Y en lo que respecta a las conductas de observación, todas las conductas tienen la frecuencia más alta, excepto el acoso sexual directo e indirecto, que tienen la frecuencia más baja.

\section{La tendencia a la agresión es masculina y a la victimización femenina}

Tal como se resume en la tabla 7, existen ciertas diferencias entre chicas y chicos respecto a sus experiencias en violencias de género 2.0. Tanto en sus experiencias como agresoras/es, víctimas o simple observadores/as.

Tabla 7

Experiencias en violencias de género 2.0 según chicas y chicos

\begin{tabular}{llllllll}
\hline \multirow{2}{*}{ Escala } & \multicolumn{1}{c}{ Dimensión } & Sexo & $\mathbf{N}$ & Media & $\begin{array}{c}\text { Desv. } \\
\text { Tìpica }\end{array}$ & t & p \\
& $\begin{array}{llllll}\text { Apartarse de la normati- } \\
\text { vidad sexual femenina }\end{array}$ & Chicos & 1571 & $\mathbf{1 , 2 2 5 5}$ &, 40962 & 2,107 &, 035 \\
& $\begin{array}{l}\text { Transgredir hetero- } \\
\text { normatividad sexual }\end{array}$ & Chicas & 1572 & $\mathbf{1 , 1 9 2 6}$ &, 45162 & & \\
& Chicos & 1472 & $\mathbf{1 , 0 6 9 7}$ &, 30689 & $-7,491$ &, 000 \\
obligatoria & & & &, 46213 & & \\
Experien- & Imposiciones del canon & Chicas & 1571 & $\mathbf{1 , 2 4 5 9}$ &, 46096 & $-4,823$ &, 000 \\
cia como & de belleza heteronor- & Chicos & 1472 & $\mathbf{1 , 3 3 7 0}$ &, 57093 & & \\
& mativo & & & & & & \\
& Violencia sexual & Chicas & 1571 & $\mathbf{1 , 0 4 4 6}$ &, 24339 & $-5,769$ &, 000 \\
& & Chicos & 1472 & $\mathbf{1 , 1 1 1 2}$ &, 37578 & & \\
& Violencia asociada a & Chicas & 1571 & $\mathbf{1 , 3 7 9 2}$ &, 62110 & 8,196 &, 000 \\
& mitos amor romántico & Chicos & 1472 & $\mathbf{1 , 2 0 9 1}$ &, 52242 & & \\
\hline
\end{tabular}




\begin{tabular}{|c|c|c|c|c|c|c|c|}
\hline Escala & Dimensión & Sexo & $\mathbf{N}$ & Media & $\begin{array}{l}\text { Desv. } \\
\text { Tìpica }\end{array}$ & $\mathbf{t}$ & $\mathbf{p}$ \\
\hline \multirow{10}{*}{$\begin{array}{l}\text { Experien- } \\
\text { cia como } \\
\text { víctima }\end{array}$} & \multirow{2}{*}{$\begin{array}{l}\text { Apartarse de la normati- } \\
\text { vidad sexual femenina }\end{array}$} & Chicas & 1571 & 1,2502 & 49193 & \multirow[t]{2}{*}{7,520} & \multirow[t]{2}{*}{,000 } \\
\hline & & Chicos & 1472 & 1,1253 & ,42284 & & \\
\hline & \multirow{2}{*}{$\begin{array}{l}\text { Transgredir hetero- } \\
\text { normatividad sexual } \\
\text { obligatoria }\end{array}$} & Chicas & 1571 & 1,0872 & ,33935 & \multirow[t]{2}{*}{$-4,488$} & \multirow[t]{2}{*}{, 000} \\
\hline & & Chicos & 1472 & 1,1527 & ,45329 & & \\
\hline & \multirow{2}{*}{$\begin{array}{l}\text { Imposiciones del canon } \\
\text { de belleza heteronor- } \\
\text { mativo }\end{array}$} & Chicas & 1571 & 1,2716 & 45543 & \multirow[t]{4}{*}{6,397} & \multirow[t]{4}{*}{000} \\
\hline & & Chicos & 1472 & 1,1668 & ,44853 & & \\
\hline & \multirow[t]{2}{*}{ Violencia sexual } & Chicas & 1571 & 1,1217 & ,38609 & & \\
\hline & & Chicos & 1472 & 1,1106 & ,39419 & & \\
\hline & \multirow{2}{*}{$\begin{array}{l}\text { Violencia asociada a } \\
\text { mitos amor romántico }\end{array}$} & Chicas & 1571 & 1,4198 & ,73996 & \multirow[t]{2}{*}{9,436} & \multirow[t]{2}{*}{,000 } \\
\hline & & Chicos & 1472 & 1,1980 &, 54771 & & \\
\hline \multirow{10}{*}{$\begin{array}{l}\text { Experien- } \\
\text { cia con ob- } \\
\text { servación }\end{array}$} & \multirow{2}{*}{$\begin{array}{l}\text { Apartarse de la normati- } \\
\text { vidad sexual femenina }\end{array}$} & Chicas & 1571 & 2,4427 & ,94459 & \multirow[t]{2}{*}{8,856} & \multirow[t]{2}{*}{, 000} \\
\hline & & Chicos & 1472 & 2,1359 & ,96482 & & \\
\hline & \multirow{2}{*}{$\begin{array}{l}\text { Transgredir hetero- } \\
\text { normatividad sexual } \\
\text { obligatoria }\end{array}$} & Chicas & 1571 & 2,2498 & ,95818 & \multirow[t]{2}{*}{4,049} & \multirow[t]{2}{*}{, 000} \\
\hline & & Chicos & 1472 & 2,1067 & ,99027 & & \\
\hline & \multirow{2}{*}{$\begin{array}{l}\text { Imposiciones del canon } \\
\text { de belleza heteronor- } \\
\text { mativo }\end{array}$} & Chicas & 1571 & 2,5594 & 97014 & \multirow[t]{2}{*}{9,887} & \multirow[t]{2}{*}{, 000} \\
\hline & & Chicos & 1472 & 2,2053 & 1,00280 & & \\
\hline & \multirow[t]{2}{*}{ Violencia sexual } & Chicas & 1571 & 1,9058 & ,91046 & \multirow[t]{2}{*}{2,374} & \multirow[t]{2}{*}{,018 } \\
\hline & & Chicos & 1472 & 1,8261 & ,93917 & & \\
\hline & \multirow{2}{*}{$\begin{array}{l}\text { Violencia asociada a } \\
\text { mitos amor romántico }\end{array}$} & Chicas & 1571 & 2,6203 & 1,08702 & \multirow[t]{2}{*}{14,779} & \multirow[t]{2}{*}{, 000} \\
\hline & & Chicos & 1472 & 2,0431 & 1,06530 & & \\
\hline
\end{tabular}

En general la tendencia a la agresión es masculina. Exceptuando los ítems: "Insultar a una chica por tener varias parejas, por ser provocativa, porque no se interesa por los chicos o porque no ha tenido relación con chicos». No obstante, en los ítems relacionados con los mitos del amor romántico las chicas muestran más conductas agresoras.

En las experiencias como víctimas, los chicos lo son cuando se apartan del modelo masculino de referencia. Las chicas son más víctimas en todas las demás dimensiones. Las chicas demuestran un nivel más elevado de consciencia a la hora de identificar todas las situaciones de violencias 2.0 que observan.

\section{Otras conductas de violencias de género 2.0}

Además de los indicadores expuestos anteriormente, llaman la atención otras tres conductas de clara violencia de género a tener en cuenta en los espacios online: «meterse con alguien por tener una ideología feminista, echar a alguien de un chat o foro por el hecho de ser mujer y enviar imágenes o hacer chistes sobre agresiones contra las mujeres». 
Los tres casos se identifican en mayor medida desde la escala de observación, y no tanto en las de agresión o victimización. Concretamente, el caso que se identifica mayoritariamente es «enviar imágenes o hacer chistes sobre agresiones contra las mujeres», seguida de «meterse con alguien por tener una ideología feminista».

Las dimensiones menos puntuadas por parte de los y las adolescentes han sido «echar a alguien de un chat o foro por el hecho de ser mujer» en las escalas de agresor y de víctima. Esta situación probablemente no sea cercana ni se dé comúnmente en adolescentes de $3 .^{\circ}$ y $4 .^{\circ}$ de ESO.

En estos indicadores se mantiene la misma tendencia anterior, tal como se resume en la tabla 8 . Los chicos puntúan más como agresores y las chicas más como víctimas y observadoras.

Tabla 8

Puntuaciones en otros indicadores de violencias de género 2.0 según sexo

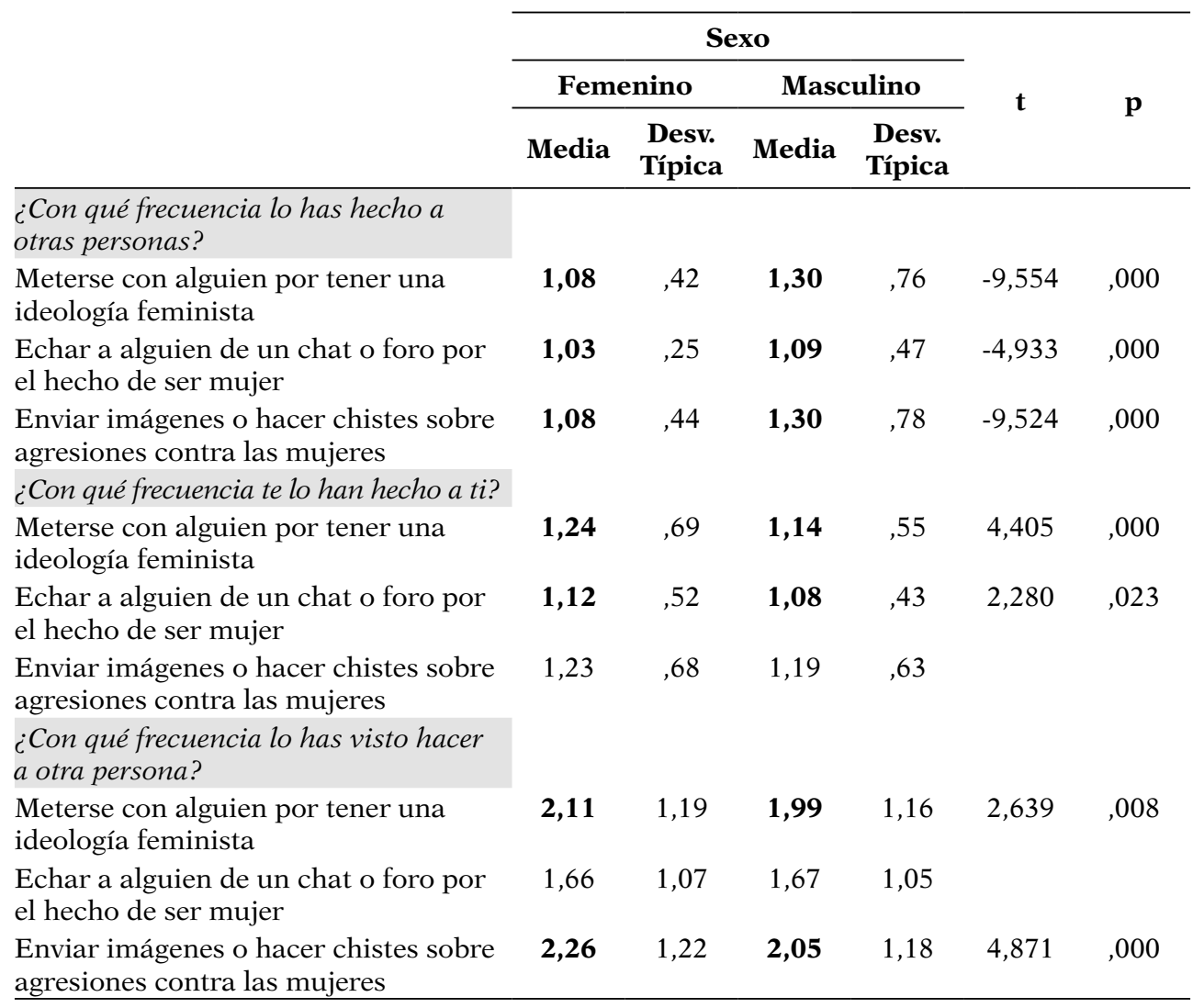


Los chicos son los que más puntúan en las tres situaciones en la escala de agresor, evidenciando que son los que más se meten con alguien por tener una ideología feminista, los que más echan a alguien de un chat o foro por el hecho de ser mujer, y los que más envían imágenes o hacen chistes sobre agresiones contra las mujeres. Las chicas son las que padecen más que se metan con ellas por tener una ideología feminista, y se las eche de un chat o foro por el hecho de ser mujer. Finalmente, las chicas son las que observan en mayor medida situaciones en su entorno relativas a meterse con alguien por tener una ideología feminista y enviar imágenes o hacer chistes sobre agresiones contra las mujeres.

\section{Respuestas ante las violencias de género 2.0}

Los y las adolescentes actúan de forma variada ante las violencias de género 2.0, como víctimas o como observadoras y observadores de las mismas (véase tabla 9). Un preocupante $24 \%$ actúa de forma pasiva cuando es víctima de estas situaciones, mientras que un $38 \%$ también actúa de forma pasiva cuando observa la violencia en otras personas.

Tabla 9

Respuestas ante las violencias de género 2.0

\begin{tabular}{llrr}
\hline & & \multicolumn{1}{c}{$\%$} \\
\hline & No me ha pasado nunca & 394 & $14,8 \%$ \\
& No he hecho nada & 646 & $24,2 \%$ \\
& Se lo he explicado a mi padre, madre, profesor/a, & 191 & $7,2 \%$ \\
& policía, .. & & \\
& Le he pedido que pare & 261 & $9,8 \%$ \\
& He cambiado de móvil, cuenta de correo o crea- & 61 & $2,3 \%$ \\
& do un perfil nuevo de Facebook & & \\
Respuestas como & He bloqueado el perfil o número de teléfono del & 905 & $33,9 \%$ \\
víctima de VG2.0 & agresor/a & 105 & $3,9 \%$ \\
& Le he hecho lo mismo & 47 & $1,8 \%$ \\
& Respuestas violentas & 13 &, $5 \%$ \\
& Respuestas dialogantes & 3 &, $1 \%$ \\
& Se lo he explicado a mis amistades & 7 &, $3 \%$ \\
& He dejado la amistad, relación, pueblo, etcétera & 33 & $1,2 \%$ \\
\hline \multirow{2}{*}{$\begin{array}{l}\text { Respuestas como } \\
\text { observador/a de }\end{array}$} & He he ignorado & 37 & $1,3 \%$ \\
VG2.0 & No he hecho nada & 1110 & $38,3 \%$ \\
& He defendido o ayudado a la persona agredida & 1709 & $58,9 \%$ \\
& He ayudado al que lo está haciendo & 44 & $1,5 \%$ \\
\hline
\end{tabular}


Respuestas cuando son víctimas de las violencias de género 2.0

Entre las respuestas más frecuentes cuando se sienten víctimas de violencias de género 2.0 observamos que el $34 \%$ bloquea el perfil o teléfono del agresor/a, y un 10\% ha pedido al agresor/a que pare. Solo el $7 \%$ lo explica a la familia $\mathrm{u}$ otras personas adultas.

Si analizamos estos datos según sean chicas o chicos (véase figura 1), se observa que los chicos están más presentes entre las respuestas pasivas (19\% de chicos frente al 14\% de chicas).

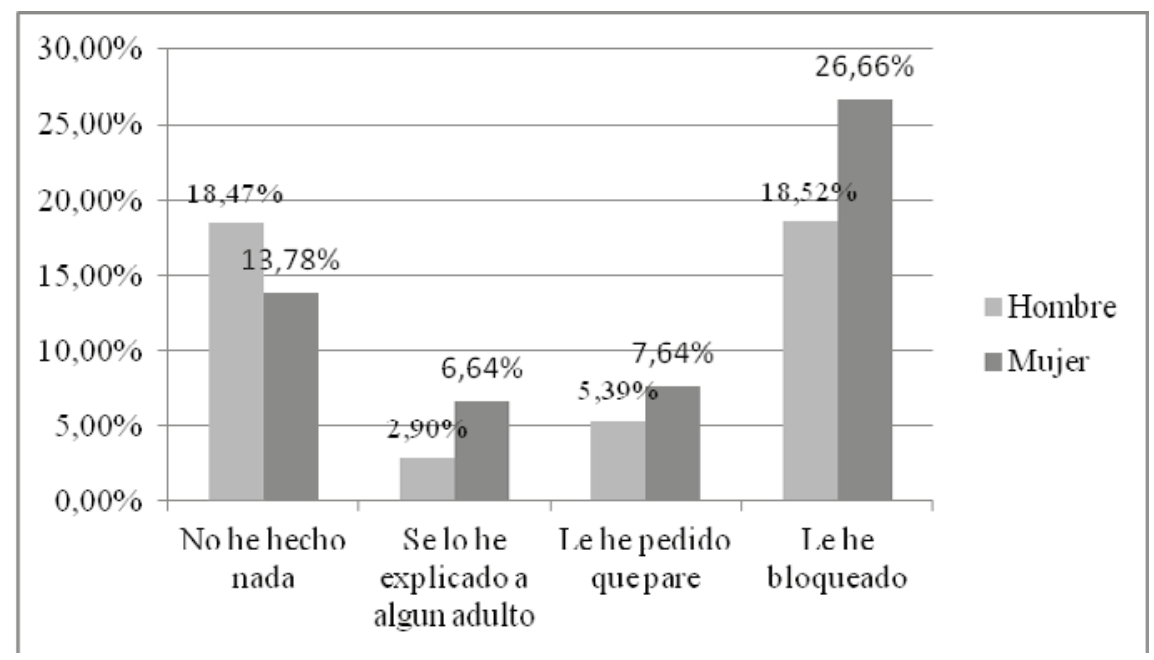

Figura 1. Respuestas ante las VG 2.0 como víctimas según sexo

Respuestas cuando se observan las violencias de género 2.0

Entre las respuestas más frecuentes cuando los y las adolescentes observan la violencia de género en su entorno online, destaca que el 59\% ayuda a la víctima y que un 38\% no interviene. De forma minoritaria, un 3\% colabora con el agresor/a ayudándole o animándolo a seguir.

Los chicos protagonizan las respuestas pasivas cuando observan algún tipo de violencia de género online: $22 \%$ de los chicos no hacen nada, ante el 17\% de las chicas. En cambio el 35\% de las chicas y el 25\% de los chicos manifiestan defender a las víctimas. 


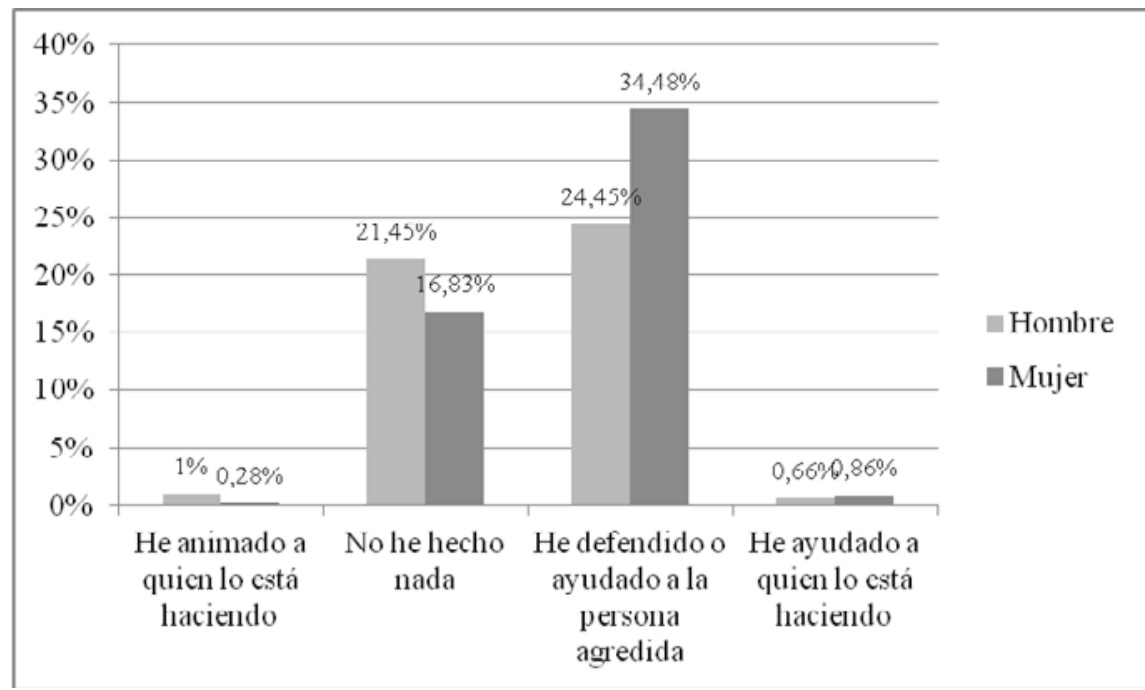

Figura 2. Respuestas ante las VG2.0 como observadores según sexo

\section{CONCLUSIONES Y DISCUSIÓN}

Los resultados encontrados en el estudio dan cuenta de los objetivos perseguidos, y son coincidentes con otras investigaciones relacionadas con temáticas de violencia en entornos virtuales.

Mayoritariamente los y las adolescentes creen que hay más violencia online que offline, una percepción significativa ya que las nuevas tecnologías forman parte de nuestro presente y las generaciones jóvenes viven, se socializan y crecen en el espacio virtual desde hace ya unos años. En la investigación de Estébanez y Vázquez (2013) los chicos y chicas reconocen que las redes sociales presentan un riesgo, por la posibilidad de usurpación de identidad, difusión de rumores e intimidades o de realizar insultos. Todo ello tiene consecuencias peores en las redes sociales, por el alcance y difusión que tiene lo que se dice o se sube en ellas.

El sistema de mensajería instantánea whatsapp y las redes youtube e instagram se usan en mayor medida. En concordancia con otros estudios, estos entornos están entre los preferidos por la población adolescente (Interctive Advertinsing Bureau, 2014 y 2015; Online Bussines School, 2015).

En las preferencias por un entorno u otro, las investigaciones continúan mostrando diferencias por sexo: comunicación y relación social priman en las chicas (hacen más comentarios, suben más fotos, cuentan más cosas personales, exponen sus sentimientos) y ocio en los chicos. Ellos, re- 
produciendo el rol masculino, prefieren no exponerse en lo sentimental, no contar demasiado, y hacer un uso más instrumental y lúdico de las redes. La expresión de sentimientos, más propia de las chicas, las convierte en más vulnerables (Gallego y García, 2011).

La percepción sobre lo que es violencia de género 2.0 se relaciona con acciones más directas y evidentes ligadas a los estereotipos de género, a la violencia sexual y a la violencia por manifestar posiciones antipatriarcales. En cambio las conductas que menos se perciben como violentas son aquellas que se ejercen contra las chicas que no se ajustan al patrón de sexualidad femenino estipulado, o aquellas en las que la mujer es tratada como objeto sexual. Según Estébanez y Vázquez (2013) en Instagram la chicas suben más autofotos que los chicos, algo que en ocasiones se considera unido a la práctica de la provocación por parte de las chicas, algo visto como propio de su género. Esta exposición pública de su imagen física hace que se expongan también a la aceptación, rechazo, o comentarios negativos por parte de los demás.

Pero sobre todo se perciben como menos violentas las conductas de control que se ejercen sobre la pareja a través de los entornos virtuales. Los datos de este último tipo de violencia están en la línea de los encontrados en el estudio de Díaz Aguado (2013) en donde se muestra cómo el control abusivo a través del teléfono móvil es el que más se ejerce entre los y las jóvenes. Este hecho se haya influenciado por los mitos románticos difundidos a través de diferentes agentes socializadores (Estébanez y Vázquez 2010; Estébanez 2010; Ferrer y Bosch, 2013). Estos sustentan y justifican las relaciones basadas en el control e intercambio de contraseñas de las redes sociales, como una prueba de amor y confianza en la pareja. En la misma línea el estudio del Ministerio de Sanidad, Servicios Sociales e Igualdad (De Miguel, 2015), evidencia que uno de cada tres jóvenes considera como inevitable controlar a la pareja y, por consecuente, se aceptan y normalizan determinadas conductas de control.

Los entornos online donde consideran los y las adolescentes que se puede ejercer más violencia son facebook, whatsapp, ask.fm e instagram. Como han demostrado otros estudios (Díaz Aguado, 2013) estas redes están entre las más utilizadas para ejercer el acoso y el control en la pareja. Ante este hecho reconocido del ejercicio de la violencia en los entornos online, los adolescentes creen que alguien debe intervenir, desde un organismo especializado hasta la familia o incluso las amistades. Y excluyen a la escuela de esta responsabilidad.

En general los y las adolescentes afirman que han observado conductas violentas en mayor medida de lo que han sido víctimas o personas 
agresoras. Es incongruente que haya un número elevado de observadores y observadoras pero que por el contrario aparezca un número bajo de víctimas y agresores/as. Parece que los y las adolescentes son más capaces de identificar las conductas de violencia cuando no son parte implicada de ellas.

La tendencia a la agresión es masculina. En el estudio de Save the children de 2010, se evidencia que las chicas se perciben más como víctimas y los chicos reconocen haber sido más perpetradores de comportamientos violentos (envío de fotos, vídeos o comentarios perjudiciales). Estas diferencias de género en cuanto a la percepción de la violencia y su tolerancia (incluyendo acoso y control virtual) fue constatada también por Sortzen Consultoría (2010) en un estudio en el que se demuestra que las chicas les asignan un sentido cariñoso o de interés por parte de la pareja cuando realiza muchas llamadas de teléfono (37\%) y los chicos lo perciben como una forma de control $(76 \%)$.

En los ítems relacionados con los mitos del amor romántico las chicas muestran más conductas agresoras, lo que se puede explicar por la apropiación por parte de estas de los comportamientos de control masculinos que históricamente se han llevado a cabo contra las mujeres y que ellas asumen como lógicos en las dinámicas relacionales. La deportación de las chicas al comportamiento masculino plantea grandes interrogantes a la acción educativa. En lugar de estar eliminándose el nivel de conductas violentas perpetradas por los chicos (Barlett y Coyne, 2014) las chicas se están sumando a este tipo de conductas.

Pero no podemos olvidar que las consecuencias de estos actos son diferentes para chicos que para chicas. Las consecuencias son más perjudiciales para las mujeres (Borrajo, Gámez y Calvete, 2015; Torres, 2013; Banyard y Cross, 2008), entre otras razones por su posición de vulnerabilidad psicológica en el entramado relacional y social.

En las experiencias como víctimas, los chicos lo son cuando se apartan del modelo masculino de referencia. Y además parecen ser más agredidos o acosados por personas de su propio sexo (Estébanez y Vázquez, 2013). Las chicas son más víctimas en todas las demás dimensiones, al tiempo que demuestran un nivel más elevado de consciencia a la hora de identificar todas las situaciones de violencias 2.0 que observan.

Las chicas reciben agresiones por tener una ideología feminista, y se las expulsa de chats y foros por el hecho de ser mujer. 
Ante las violencias de género 2.0 una parte de la muestra actúa de forma pasiva cuando es víctima de estas situaciones, o cuando observa la violencia en otras personas. Los chicos están más presentes entre las respuestas pasivas y las chicas superan a los chicos en las respuestas activas frente a la violencia. Con mayor frecuencia las chicas explican a la familia, profesorado o policía sus experiencias; piden que paren y bloquean el perfil o teléfono del agresor cuando son cibervíctimas. Al mismo tiempo, las chicas cuando observan violencias de género 2.0 defienden o ayudan a la persona agredida más a menudo que los chicos.

\section{IMPLICACIONES EDUCATIVAS}

La investigación ha evidenciado que los y las adolescentes presentan concepciones y comportamientos inadecuados que pueden contribuir a aumentar su vulnerabilidad ante las violencias de género 2.0. Para eliminar estas concepciones y comportamientos se precisan acciones educativas implicando en ellas a la escuela. Este agente aducativo no está contemplado por los adolescentes como mediador en los conflictos derivados de la violencia de género 2.0, un hecho que atribuimos a la falta de preparación del profesorado en esta temática. Por ello consideramos que las acciones deben dirigirse tanto al alumnado como al profesorado.

El profesorado debe conocer la existencia y prevalencia del fenómeno en sus distintas dimensiones, así como sus consecuencias, y también debe recibir formación relacionada con las acciones que puede emprender como agente educativo y mediador de conflictos.

Por su parte, el alumnado debe recibir formación que ayude a protegerlo del fenómeno de la violencia de género 2.0, una formación orientada a: comprender el concepto en toda su amplitud y fomentar una actitud crítica hacia el mismo, deconstruir los mitos del amor romántico que sustentan falsas ideas sobre la confianza en la pareja, evidenciar las amenazas que pueden encontrar en los entornos virtuales, concienciar a las chicas sobre su mayor vulnerabilidad en las redes sociales, explicar a los y las adolescentes los malos usos de las redes sociales y sus riesgos derivados, y alentarles a denunciar las acciones de violencia implicando a la familia y la escuela en estos asuntos.

Las acciones educativas pueden adquirir diferentes formatos, desde talleres informativos hasta acciones más participativas que impliquen al alumnado en su aprendizaje y responsabilidad en relación al fenómeno de la violencia de género en los entornos virtuales. En el taller de Prado 
(2015) o el de Prado y Vilà (2015) pueden comprobarse aspectos necesarios a desarrollar con relación a la problemática de la violencia que se ejerce en los entornos virtuales sobre las mujeres y otros grupos de personas (homosexuales, transexuales...) a causa de la desigualdad de géneros estipulada desde el sistema patriarcal, con los siguientes objetivos educativos:

- Generar espíritu crítico del grupo contra la violencia de género.

- Poner de manifiesto las diferencias y similitudes entre la violencia de género offline y online.

- Dar a conocer los diferentes tipos de violencia de género que tienen lugar en espacios virtuales.

- Informar sobre los posibles riesgos que los y las adolescentes pueden encontrarse en los entornos online.

- Fomentar buenos hábitos de uso de las redes sociales, aplicaciones y entornos virtuales.

- Concienciar sobre la importancia de utilizar una net-etiqueta desde la perspectiva de género con el fin de mejorar la utilización de espacios online.

- Ofrecer recomendaciones para mejorar la navegación de una forma más segura.

- Promover recursos e iniciativas online que luchan contra la violencia de género.

El taller se centra en contenidos sobre el mal uso de las tecnologías desde la perspectiva de género, poniendo de manifiesto que este es un reflejo de las relaciones que se dan fuera de ellas, en el ámbito offline. El taller va dirigido a adolescentes y se basa en sus percepciones sobre algunas herramientas, comportamientos, mitos y creencias sobre la violencia online que se ejerce y se padece en base al género, y se propone poner de manifiesto las amenazas que pueden encontrarse en los entornos virtuales, pero también fomentar una actitud crítica sobre este tipo de violencias, así como un buen uso de los medios a su alcance para frenar cualquier tipo de agresión (Prado y Vilà, 2015). 


\section{REFERENCIAS BIBLIOGRÁFICAS}

Arisó, O. y Mérida, R. (2010). Los géneros de la violencia Una reflexión queer sobre la violencia de género. Barcelona: Egales.

Asociación para la Investigación de Medios de Comunicación (2013). Resumen general de resultados. Febrero a Noviembre de 2013. Recuperado de http://bit.ly/1eKM3M0

Banyard, V. L. y Cross, C. (2008). Consequences of teen dating violence understanding intervening variables in ecological context. Violence Against Women, 14(9), 998-1013.

Barlett, C. \& Coyne, S. M. (2014). A meta-analysis of sex differences in cyber-bullying behavior: The moderating role of age. Aggressive Behavior, 40(5), 474-488.

Borrajo, E., Gámez, M. \& Calvete, E. (2015). Justification beliefs of violence, myths about love and cyber dating abuse. Psicothema, 27(4), 327-333. doi: 10.7334/psicothema2015.59

Calmaestra, J. (2011). Cyberbullying: prevalencia y características de un nuevo tipo de bullying indirecto [Tesis Doctoral]. Servicio de Publicaciones de la Universidad de Córdoba.

Calvete, E.; Orue, I.; Estévez, A.; Villardón, L. \& Padilla, P. (2010). Cyberbullying in adolescents: Modalities and aggressors' profile. Computers in Human Behavior, 26, 1128-1135. doi:10.1016/j.chb.2010.03.017

Díaz-Aguado, M. J (2013). La evolución de la adolescencia española sobre la igualdad y prevención de la violencia de género. Madrid: Ministerio de Sanidad, Servicios Sociales e Igualdad. Recuperado de http://bit.ly/2knE4Ru
De Miguel, V. (2015). Percepción social de la violencia de género en la adolescencia y la juventud. Madrid: Ministerio de Sanidad, Servicios Sociales e Igualdad. Recuperado de http://bit.ly/1tkldqj

Donoso, T; Rubio, M. J; Velasco, A. y Vilà, R. (2014a). Cuestionario de violencias de género 2.0. Barcelona: Universitat de Barcelona.

- (2014b). Ciberacoso en función del género. Propuetas de análisis. $V$ Congreso Universitario Internacional de Investigación y Género. Sevilla: Universidad de Sevilla.

Donoso, T; Rubio, M. J. y Vilà, R. (2017). Las ciberagresiones en función del género. Revista de Investigación Educativa, 35(1), 197-214.

Estébanez, I. (2010). Te quiero... (sólo para mí). Relaciones adolescentes de control. Revista pedagógica, 23, 45-68. Recuperado de http://bit.ly/1uItFhJ

Estébanez, I. \& Vázquez, N. (2010). Diagnóstico de la percepción y opiniones sobre la violencia sexista de la juventud de los municipios de Ondarroa y Markina-Xemein. Bilbao: Sortzen Consultoría. Recuperado de http://bit. ly/2ko9jfc

- (2013). La desigualdad de género y el sexismo en las redes sociales. Bilbao: Observatorio Vasco de la Juventud.

Ferrer, V. y Bosch, E. (2013) Del amor romántico a la violencia de género. Para una coeducación emocional en la agenda educativa. Profesorado. Revista de curriculum y formación del profesorado, 17(1), 105-122.

Gallego, M. y García, L. (2011). Violencia basada en género en las relaciones de pareja. Recuperado de http://bit. $\mathrm{ly} / 2 \mathrm{jrCzSS}$ 
García Ramos, T. N. (s.f.). e-women. Informe sobre la Violencia de Género en Internet. Recuperado de http://bit. ly/2jzAyPG

Garmendia, M; Garitaonandia, C; Martínez, G. y Casado, M. A. (2011). EU Kids Online II: Mejorando el conocimiento sobre el uso y la seguridad en Internet de los menores en Europa. Recuperado de https://goo.gl/tBChHv

Interctive Advertinsing Bureau (2015). VI Estudio sobre Redes Sociales. Recuperado de http://bit.ly/18tGiW7

Lagarde, M. (1997) Género y Feminismo. Desarrollo Humano y Democracia. Madrid: Edición Horas y Horas.

Li, Q. (2007). New bottle but old wine: a research of cyberbullying in schools. Computers in Human Behavior 23 (4), 1777-1791. doi:10.1016/j.chb. 2005.10.005.

Online Business School (2015). Social media 2015: Análisis de las tendencias de uso y participación en las redes social a nivel mundial y en España.

Prado, N. (2015). Taller sobre violencias de género 2.0. Barcelona: Universidad de Barcelona. Recuperado de http:// hdl.handle.net/2445/67141

Prado, N. y Vilà, R. (2015). Taller sobre Violencias de Género 2.0. Diseño. Barcelona: niversidad de Barcelona.
Recuperado de http://hdl.handle. net/2445/67493

Patchin, J. W. \& Hinduja, S. (2006). Bullies move beyond the schoolyard: A preliminary look atcyberbullying. Youth Violence and Juvenile Justice, 4(2), 148-169.

Save the children (2010). La tecnología en la preadolescencia y adolescencia: usos, riesgos y propuestas desde los protagonistas. Recuperado de http:// bit.ly/1pegqSq

Sortzen Consultoría (2010). Diagnóstico de la percepción y opiniones sobre la violencia sexista de la juventud de los municipios de Ondarroa y Markina-Xemein. Bilbao: Sortzen Consultoría.

Torres, C. (2013). El ciberacoso como forma de ejercer la violencia de género en la juventud: un riesgo en la sociedad de la información y del conocimiento. Madrid: Delegación del Gobierno para la Violencia de Género.

Unión Internacional de Telecomunicaciones (2013). Medición de la Sociedad de la Información. Resumen ejecutivo. Ginebra: Unión Internacional de Telecomunicaciones. Recuperado de http://bit.ly/1pNAk8t

Working to Halt Online Abuse. (2012). Online harassment statistics. WHOA. Recuperado de http://bit.ly/2jS1zir 


\section{PERFIL ACADÉMICO Y PROFESIONAL DE LOS AUTORES}

Trinidad Donoso Vázquez. Profesora titular del Departamento de Métodos de Investigación y Diagnóstico en Educación de la Universidad de Barcelona. Ha sido delegada del rector para los temas de igualdad de género en la Universidad de Barcelona. Entre sus últimos proyectos cabe destacar el diseño y elaboración de programas de Intervención en Violencias de Género, la evaluación de los mismos y los procesos de construcción de una ciudadanía paritaria. Coordina la línea de investigación GrediDona.

M. José Rubio Hurtado. Profesora titular del departamento de Métodos de Investigación y Diagnóstico en Educación de la Universidad de Barcelona. Investigadora de los grupos de investigación GREAV (Enseñanza y aprendizaje en entornos virtuales) y GediDona, ha dirigido y participado en estudios de género y TIC, y en estudios relacionados con los nuevos entornos digitales aplicados a la educación.

Ruth Vilà Baño. Profesora del Departamento de Métodos de Investigación y Diagnóstico en Educación de la Universidad de Barcelona. Miembro del grupo de Investigación GrediDona. Ha dirigido la investigación «Violencias de género 2.0» financiada por la Fundación BBVA. Es formadora y asesora metodológica en el Instituto de Ciencias de la Educación (ICE) de la Universidad de Barcelona y también imparte docencia en diversos Másteres y Postgrados de otras universidades.

Dirección de los autores: Universidad de Barcelona

Facultad de Educación

Campus Vall d'Hebron, 171

08035 Barcelona

E-mail: trinydonoso@ub.edu

mjrubio@ub.edu

ruth_vila@ub.edu

Fecha modificación artículo: 21. Julio. 2016

Fecha recepción del artículo: 04. Febrero. 2016

Fecha aceptación del artículo: 06. Septiembre. 2016

Fecha revisión para publicación: 31. Mayo. 2017 
